\title{
Indicators for prediction of Mycobacterium tuberculosis positivity detected with bronchoalveolar lavage fluid
}

Xi Liu' ${ }^{1,2}$, Xing-Fang Hou' ${ }^{1}$, Lei Gao ${ }^{3}$, Guo-Fang Deng ${ }^{1}$, Ming-Xia Zhang ${ }^{1}$, Qun-Yi Deng ${ }^{1}$, Tao-Sheng Ye ${ }^{1}$, Qian-Ting Yang ${ }^{1}$, Bo-Ping Zhou' ${ }^{1}$ Zhi-Hua Wen ${ }^{5}$, Hai-Ying Liu ${ }^{3}$, Hardy Kornfeld ${ }^{4}$ and Xin-Chun Chen ${ }^{1,6^{*}}$

\begin{abstract}
Background: The diagnosis of active pulmonary tuberculosis (TB) remains a challenge in clinic, especially for sputum negative pulmonary TB. Bronchoalveolar lavage fluid (BALF) has higher sensitivity than sputum for detection of Mycobacterium tuberculosis (Mtb). However, bronchoscopy is invasive and costly, and not suitable for all patients. In order to make TB patients get more benefit from BALF for diagnosis, we explore which indicator might be used to optimize the choice of bronchoscopy.

Methods: A total of 1539 sputum-smear-negative pulmonary TB suspects who underwent bronchoscopy were recruited for evaluation. The sensitivity, specificity and accuracy of Mtb detection in sputum and BALF were compared. Odds ratios and $95 \%$ confidence intervals were used to assess variables that associated with positive acid-fast bacilli (AFB) smear, Mtb culture and nucleic acid amplification test (NAAT) of BALF in sputum-negative and non-sputum-producing pulmonary TB suspects.

Results: BALF has significantly higher sensitivity (63.4\%) than sputum (43.5\%) for Mtb detection by culture and NAAT. 19.7\% (122/620) sputum-negative and 40.0\% (163/408) non-sputum-producing suspects had positive bacteriological results in BALF. Among sputum-negative and non-sputum-producing pulmonary TB suspects, the positivity of Mtb detection in BALF is associated with a younger age, the presence of pulmonary cavities and a positive result of interferon-gamma release assay (IGRA). Sputum-negative patients under 35 years old with positive IGRA and pulmonary cavity had $84.8 \%$ positivity of Mtb in BALF.

Conclusions: Our study indicated that combination of age, the presence of pulmonary cavity, and the result of IGRA is useful to predict the positivity of Mtb detection in BALF among sputum-negative and non-sputum producing pulmonary TB suspects. Those who are under 35 years old, positive for the presence of pulmonary cavity and IGRA, should undergo bronchoscopy to collect BAFL for Mtb tests, as they have the highest possibility to get bacteriologically confirmation of TB.
\end{abstract}

Keywords: Pulmonary tuberculosis, Predictive factors, Bronchoalveolar lavage fluid, Mycobacterium tuberculosis, Detection

\footnotetext{
* Correspondence: chenxinchun@szu.edu.cn

${ }^{1}$ Shenzhen Key Laboratory of Infection \& Immunity, Shenzhen Third People's

Hospital (The Second Affiliated Hospital of Shenzhen University), Shenzhen

University School of Medicine, Shenzhen, China

${ }^{6}$ Department of Pathogen Biology, Shenzhen University School of Medicine,

Shenzhen 518054, China

Full list of author information is available at the end of the article
} 


\section{Multilingual abstracts}

Please see Additional file 1 for translations of the abstract into the five official working languages of the United Nations.

\section{Background}

Tuberculosis (TB), an airborne infection caused by Mycobacterium tuberculosis (Mtb), remains a major global health problem. In 2015, there were estimated 10.4 million new TB cases, and 1.8 million TB deaths. Despite this large toll of mortality, majority of TB patients can be cured within 6 months with timely diagnosis and correct treatment [1]. Considering there is no effective preventive vaccine against $\mathrm{TB}$, the early diagnosis of pulmonary $\mathrm{TB}$ patients is not only critical for individual treatment, but also a major strategy for prevention of TB transmission. Microbiological confirmation of TB and drug susceptibility testing are key factors ensuring that new TB suspects are accurately diagnosed and timely accessible to effective treatment. Unfortunately, it remains as a challenge as up to $65 \%$ of $\mathrm{TB}$ patients were negative for Mtb detection in China [1]. There is a clear necessity to identity optimal methods for pulmonary TB diagnosis of bacteriologicallynegative patients, particularly in high burden settings of multi-drug resistant TB (MDR-TB) like China.

Newer methods with increased sensitivity compared to traditional acid-fast bacilli (AFB) smear and culture have been developed during the last decades [2]. For example, molecular DNA-based diagnostics such as Xpert MTB/ RIF [3] and line probe assays (LPAs) [4], have become widely available and permit both rapid diagnosis and preliminary assessment of drug susceptibility [5]. While by smear, culture and nucleic acid amplification test (NAATs) have made an important contribution by accelerating TB diagnosis and preliminary identification of MDR-TB, the sensitivity remains too low [6]. Consequently, a substantial proportion of pulmonary TB patients are diagnosed based only on symptoms, chest X-ray abnormalities or (rarely) characteristic histopathology [7]. In China, the positive rate of bacteriologically confirmed pulmonary TB is only $35 \%$ among 1.38 million pulmonary TB cases [1]. Among these patients, some were bacteriologically negative due to lack of sputum or false-negative due to poor sputum sample quality. Bronchoscopy has demonstrated superiority to expectorated sputum for pulmonary TB diagnosis. As an example, a recent study reported that $54 \%$ of sputumnegative pulmonary TB patients were eventually confirmed by finding Mtb in BALF samples by NAAT or culture [8].

Although BALF offers the potential to improve the positivity of microbiological finding for diagnosis of pulmonary $\mathrm{TB}$, bronchoscopy is invasive and costly. In order to maximize the benefit of bronchoscopy while minimizing the cost to TB control programs, it is important to identify parameters to predict the yield of BALF for diagnosis of sputum- negative pulmonary TB [9]. In the present study, we compared the sensitivity and specificity of Mtb tests between sputum and BALF for diagnosis of pulmonary TB among 1539 sputum-smear-negative cases, and identified clinical parameters which are associated with the benefit of bronchoscopy in sputum-negative and nonsputum-producing pulmonary TB suspects.

\section{Methods \\ Study population}

This retrospective study was conducted at Shenzhen Third People's Hospital (Guangdong, China). Between February 2011 and May 2015, a total of 1539 sputumsmear-negative pulmonary TB suspects who underwent bronchoscopy for medical diagnosis purpose were included in the study. The selection of TB suspects for bronchoscopy was made at the discretion of the clinic physicians responsible for their care with no influence from the investigators implementing this study. Medical data were collected on age, gender, symptoms, previous TB history, laboratory test and imaging detection. Laboratory tests included sputum smear (three times) and culture, interferon-gamma release assay (IGRA), CD4 and CD8 cell counts. Patients with a follow-up less than 6 months were excluded.

\section{Cases definition}

The diagnosis of active TB were following Chinese diagnostic criteria for tuberculosis (WS288-2008), WHO guidelines for treatment of tuberculosis (fourth edition), and other references [10-12]. Active TB cases were classified as definite $\mathrm{TB}$ and probable $\mathrm{TB}$, the definition and category of study population were listed below:

Tuberculosis suspect referred to any person who presented with typical chest imaging suggestive of TB (WS288-2008).

Definite TB was defined as sputum or BALF culture positive for Mtb, or NAAT positive for Mtb, plus clinical symptom and CT sign suggestive of pulmonary TB and response to anti-tuberculosis treatment.

Probable TB was defined as sputum or BALF culture and NAAT negative for Mtb, sputum/BALF negative or positive for AFB, plus clinical symptom and CT sign suggestive of pulmonary $\mathrm{TB}$ and response to antituberculosis treatment.

Cured TB (RxTB) was defined as the pulmonary TB patients who had completed standard anti-TB treatment regimen for at least 6 months with no subsequent evidence of active TB.

Non-TB lung disease (non-TB) was defined as negative cultures and smears for Mtb detection in sputum and BALF, CT suggestive of non-TB, and anti-TB treatment was never initiated by healthcare providers. Non-TB 


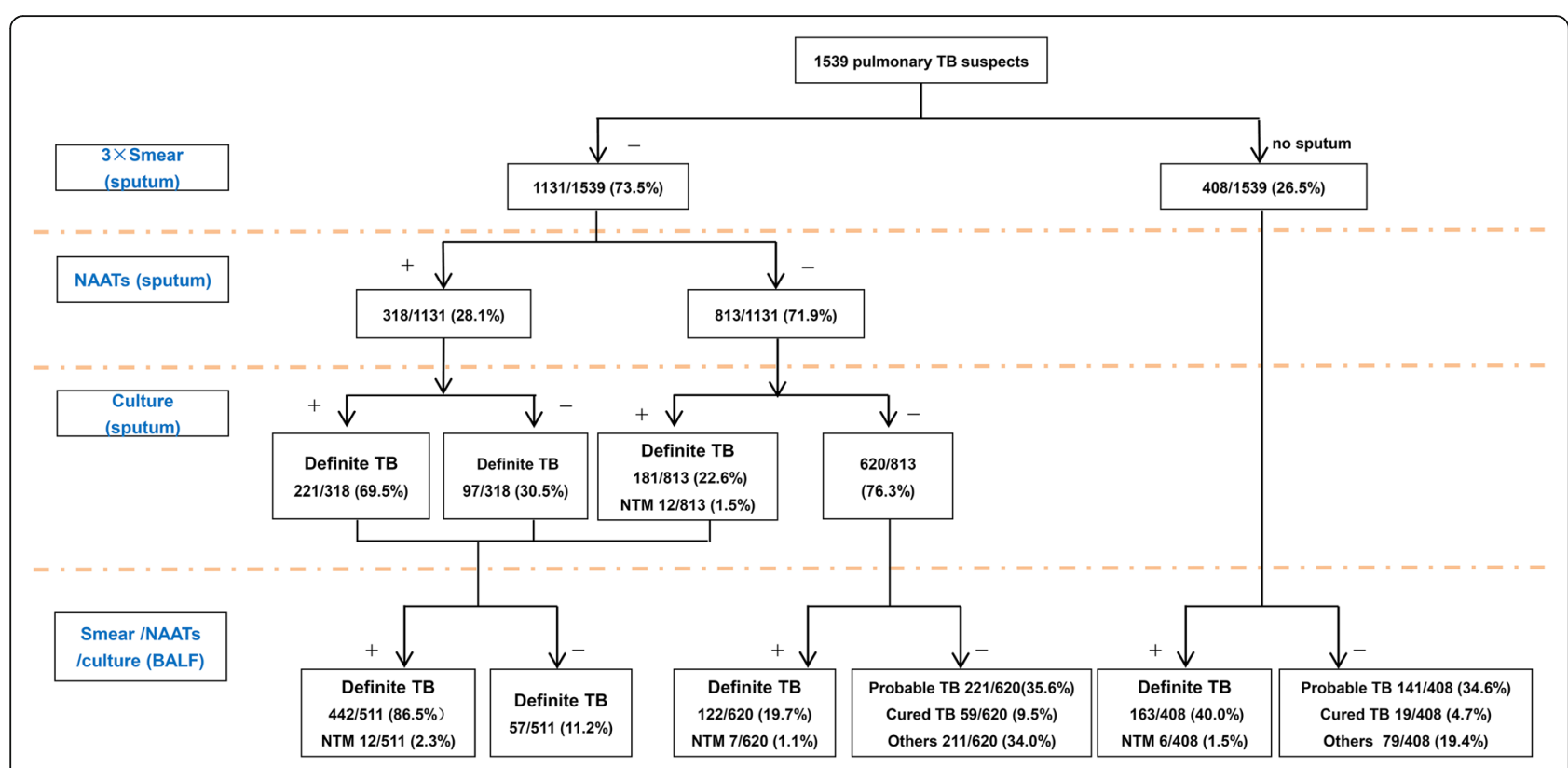

Fig. 1 Diagnostic flow chart. Abbreviations: NTM, nontuberculosis mycobateria; NAAT, nucleic acid amplification technique; BALF, bronchoalveolar lavage fluid

lung diseases included bacterial pneumonia, lung cancer, sarcoidosis, asthma, bronchiectasis, bronchitis, etc.

Non-tuberculous mycobacteria (NTM) infection was confirmed by identification of the same species of NTM in sputum and/or BALF.

\section{Bronchoscopy technique, specimen processing and IGRA}

All patients underwent bronchoscopy with BALF collection. Patients were pre-medicated with $0.5 \mathrm{mg}$ atropine and then sedated with midazolam. Bronchial washing of the involved sub-segment was performed with 20 to $50 \mathrm{ml}$ of isotonic saline. Sputum samples, if available, were collected on three consecutive days and examined for the presence of acid-fast bacilli by microscopy. All sputum and BALF samples were subjected to NAAT for detection of Mtb DNA using a commercial quantitative real-time polymerase chain reaction kit (qPCR) (Qiagen, China), and mycobacterium culture for up to 6 weeks in fluid media (MGIT, BD, Heidelberg), or 8 weeks in solid media (Loewenstein-Jensen), before the results were concluded negative.

The Mtb-specific IGRA was performed as an inhouse IFN- $\gamma$ enzyme-linked immunospot assay which was reported previously [13]. The overall sensitivities and specificities of our in-house IGRA were similar to commercial IGRA kits [13].

\section{Statistical analysis}

The positive rate, sensitivity, specificity and accuracy of Mtb tests in sputum and BALF for the diagnosis of pulmonary TB were calculated.
For categorical variables, the percentage of patients in each category was calculated. To identify potential variables related to positivity of BALF (positivity in Mtb detection of BALF through smear microscopy, culture and/ or NAAT), univariate analysis with Pearson's $\chi^{2}$ test was performed. All variables with $P$ values less than 0.05 in univariate analysis were entered into the unconditional multiple logistic regression analyses and the associations assessed with Odds ratios (OR) and 95\% confidence intervals (CIs). To identify the overlapping effects on positivity of BALF, variables with $P$ values less than 0.05 in unconditional multiple logistic regression analyses were entered into stratified analysis. All analyses were performed using SPSS software (release 20.0, IBM,USA).

Table 1 Clinical characters of pulmonary TB suspects who underwent bronchoscopy

\begin{tabular}{|c|c|c|c|}
\hline Final diagnosis & Number & Age $($ mean $\pm S D)$ & Male (\%) \\
\hline Active pulmonary TB & 1146 & $35.0 \pm 13.5$ & $636(55.5 \%)$ \\
\hline Cured tuberculosis & 78 & $42.9 \pm 13.3$ & $51(65.4 \%)$ \\
\hline NTM & 25 & $45.5 \pm 15.9$ & $8(32.0 \%)$ \\
\hline Other lung disease & 290 & $41.4 \pm 15.1$ & $170(58.6 \%)$ \\
\hline Pneumonia & 193 & $40.9 \pm 15.9$ & $111(57.5 \%)$ \\
\hline Lung cancer & 34 & $49.8 \pm 13.9$ & $21(61.8 \%)$ \\
\hline Others $^{a}$ & 62 & $38.4 \pm 11.5$ & $38(61.3 \%)$ \\
\hline Total & 1539 & $36.8 \pm 13.9$ & $865(56.2 \%)$ \\
\hline
\end{tabular}

TB tuberculosis, NTM nontuberculosis mycobateria

a Others included sarcoidosis, asthma, bronchiectasis, bronchitis, etc 
Table 2 Comparison of sensitivity, specificity, etc. between sputum and BALF for the diagnosis of pulmonary TB in sputum-smearnegative suspects

\begin{tabular}{lllll}
\hline & Positive detectable rate (\%) & Sensitivity (\%) & Specificity (\%) & Accuracy (\%) \\
\hline Sputum-culture/NAAT & 32.4 & 43.5 & 100.0 & 58.0 \\
sputum-culture & 26.1 & 35.1 & 100.0 & 51.7 \\
sputum-NAAT & 20.7 & 27.7 & 100.0 & 46.2 \\
BALF-smear/culture/NAAT & 47.2 & 63.4 & 100.0 & 72.8 \\
BALF-smear & 9.2 & 12.0 & 99.2 & 34.3 \\
BALF-culture & 42.5 & 57.1 & 100.0 & 68.0 \\
BALF-NAAT & 33.2 & 44.5 & 99.7 & 58.6 \\
Sputum+BALF & 50.9 & 68.4 & 100.0 & 72.8 \\
\hline
\end{tabular}

TB tuberculosis, BALF bronchoalveolar lavage fluid, NAAT nucleic acid amplification test

\section{Results}

Clinical characteristics and final diagnosis of sputumsmear-negative pulmonary TB suspects

A total of 1539 subjects who underwent bronchoscopy were enrolled in the study (see Fig. 1). The median age was $36.8 \pm 13.9$ (interquartile range, 11 to 81 years old) and $56.2 \%$ were males. There were no complications from the procedure and BALF samples were obtained from all patients. Among them, 1146 patients had a final diagnosis of pulmonary TB, 78 patients were cured TB but not TB relapse, 25 patients were diagnosed with NTM infection, and 290 patients had a final diagnosis of non-TB lung diseases (see Table 1).

Among 1539 suspect TB patients, 620 (40.3\%) patients were negative for sputum Mtb culture or NAAT test, 408 (26.5\%) patients had no sputum. Of 1146 suspects with final diagnosis of pulmonary TB, 304 (26.5\%) had no sputum production, 343 (29.9\%) were negative for sputum Mtb detection by smear, culture or NAAT, while 499 (43.5\%) were bacteriologically confirmed based on one or more of these sputum tests. In comparison, 727 of 1146
(63.4\%) pulmonary TB patients were bacteriologically confirmed by smear, culture or NAAT in BALF. A total of $362(31.6 \%)$ patients had a final clinical diagnosis of pulmonary TB based on chest imaging and anti-TB treatment response.

Among 25 NTM lung disease cases who were diagnosed by conventional mycobacterial culture and 16 s RNA gene based molecular species identification using BALF samples, only $48.0 \%(12 / 25)$ cases were identified by sputum culture. Bronchoscopy made more species identification possible, from which a correct treatment could be applied.

BALF improved the diagnostic accuracy of sputumnegative and non-sputum-producing TB suspects

Positive detectable rate, sensitivity, specificity and accuracy of different methods for the diagnosis of all sputumsmear-negative pulmonary TB suspects, sputum negative and no sputum patients were listed in Tables 2 and 3 . Among the 1539 suspects, the overall positive detection rate of BALF for diagnosis of pulmonary TB was significantly higher than that of sputum, with overall positivity

Table 3 The performance of BALF based detection for the diagnosis of pulmonary TB in sputum-negative suspects and those without sputum

\begin{tabular}{|c|c|c|c|c|}
\hline & Positive detectable rate $\%$ & Sensitivity \% & Specificity \% & Accuracy $\%$ \\
\hline \multicolumn{5}{|l|}{ Sputum-negative } \\
\hline BALF-smear/culture/NAAT & 19.7 & 35.6 & 100.0 & 64.4 \\
\hline BALF-culture & 16.5 & 29.7 & 100.0 & 61.1 \\
\hline BALF-NAAT & 11.1 & 20.1 & 100.0 & 55.8 \\
\hline \multicolumn{5}{|l|}{ No sputum } \\
\hline BALF-smear/culture/NAAT & 40.0 & 53.6 & 100.0 & 65.4 \\
\hline BALF-culture & 38.2 & 51.3 & 100.0 & 63.7 \\
\hline BALF-NAAT & 24.5 & 32.9 & 100.0 & 50.0 \\
\hline \multicolumn{5}{|c|}{ sputum negative and no sputum } \\
\hline BALF-smear/culture/NAAT & 27.8 & 44.1 & 100.0 & 64.9 \\
\hline BALF-culture & 26.3 & 41.6 & 100.0 & 63.3 \\
\hline BALF-NAAT & 16.5 & 26.3 & 100.0 & 53.7 \\
\hline
\end{tabular}

TB tuberculosis, BALF bronchoalveolar lavage fluid, NAAT nucleic acid amplification test 
Table 4 Comparison of clinical characteristics between BALF bacteriologically negative and positive patients within sputum negative pulmonary TB patients and those without sputum

\begin{tabular}{|c|c|c|c|c|c|}
\hline & Total & BALF (+) & BALF (-) & $P$-value & OR $(95 \% C l)$ \\
\hline & $N=1028$ & $n=285$ & $n=743$ & & \\
\hline$\overline{\text { Age }}$ & $37.4 \pm 14.2$ & $32.7 \pm 12.8$ & $39.3 \pm 14.2$ & $<0.001$ & \\
\hline Male & 589 & 154 (26.1\%) & 435 (73.9\%) & 0.191 & $0.832(0.632 \sim 1.096)$ \\
\hline TB history & 126 & $40(31.7 \%)$ & $86(68.3 \%)$ & 0.282 & $1.247(0.834-1.866)$ \\
\hline Fever & 363 & 94 (25.9\%) & $269(74.1 \%)$ & 0.333 & $0.867(0.650-1.157)$ \\
\hline Night sweat & 97 & $28(28.9 \%)$ & $69(71.1 \%)$ & 0.792 & $1.064(0.670-1.689)$ \\
\hline Cough & 751 & $213(28.4 \%)$ & $538(71.6 \%)$ & 0.451 & $1.127(0.825-1.540)$ \\
\hline Hemoptysis & 158 & 60 (38.0\%) & $98(62.0 \%)$ & 0.002 & $1.755(1.230-2.504)$ \\
\hline Lose weight & 222 & $72(32.4 \%)$ & $150(67.6 \%)$ & 0.077 & $1.336(0.969-1.843)$ \\
\hline Bilateral lung & 268 & $92(34.3 \%)$ & $176(65.7 \%)$ & 0.005 & $1.536(1.137-2.074)$ \\
\hline Cavity & 104 & $57(54.8 \%)$ & $47(45.2 \%)$ & $<0.001$ & $4.108(2.376-7.102)$ \\
\hline${ }^{\mathrm{a}} \mathrm{CD} 4$ & $595.8 \pm 274.4$ & $547.77 \pm 246.14$ & $615.8 \pm 283.2$ & 0.004 & \\
\hline${ }^{\mathrm{a}} \mathrm{CD} 4 \leq 500$ & 264 & 92 (34.8\%) & $172(65.2 \%)$ & 0.011 & $1.540(1.101-2.153)$ \\
\hline${ }^{\mathrm{a}} \mathrm{CD} 8$ & $420.0 \pm 205.4$ & $380.30 \pm 181.83$ & $436.7 \pm 212.6$ & 0.001 & \\
\hline${ }^{\mathrm{a}} \mathrm{CD} 8 \leq 500$ & 468 & $153(32.7 \%)$ & 315 (67.3\%) & 0.002 & $1.992(1.289-3.076)$ \\
\hline ESR & $40.9 \pm 31.3$ & $46.08 \pm 29.97$ & $39.0 \pm 31.6$ & 0.001 & \\
\hline $\operatorname{IGRA}(+)^{\mathrm{b}}$ & 507 & $201(39.6 \%)$ & $306(60.4 \%)$ & $<0.001$ & $3.743(2.478-5.655)$ \\
\hline
\end{tabular}

$B A L F$ bronchoalveolar lavage fluid, IGRA interferon- $\gamma$ release assays, ESR erythrocyte sedimentation rate

${ }^{\text {a Total } N=679, \operatorname{BALF}(+)} n=199, \operatorname{BALF}(-) n=480$

${ }^{\mathrm{b}}$ Total $N=938, \operatorname{BALF}(+) n=270, \operatorname{BALF}(-) n=692$

Table 5 Univariate and multivariate analysis of factors associated with BALF positivity in pulmonary TB sputum-negative suspects and those without sputum

\begin{tabular}{|c|c|c|c|c|c|}
\hline \multirow{2}{*}{\multicolumn{2}{|c|}{ Total }} & \multirow{2}{*}{$\begin{array}{l}\operatorname{BALF}(+) \\
n=285(\%)\end{array}$} & \multirow{2}{*}{$\begin{array}{l}\text { BALF (-) } \\
n=743(\%)\end{array}$} & \multirow[t]{2}{*}{$P$-value } & \multirow[t]{2}{*}{$O R(95 \% C l)^{\mathrm{a}}$} \\
\hline & & & & & \\
\hline Age (year) & & & & $<0.001$ & \\
\hline$\leq 25$ & 244 & $105(43.0)$ & $139(57.0)$ & & Reference \\
\hline $25-35$ & 288 & 88 (30.6) & $200(69.4)$ & & $0.582(0.408-0.832)$ \\
\hline $35-45$ & 215 & $44(20.5)$ & $171(79.5)$ & & $0.341(0.224-0.517)$ \\
\hline $45-55$ & 128 & $22(17.2)$ & $116(82.8)$ & & $0.251(0.149-0.423)$ \\
\hline $55-65$ & 106 & $23(21.7)$ & $83(78.3)$ & & $0.367(0.217-0.621)$ \\
\hline$>65$ & 37 & $3(8.1)$ & $34(91.9)$ & & $0.117(0.035-0.391)$ \\
\hline Cavity & & & & $<0.001$ & \\
\hline No & 924 & $228(24.7)$ & $696(75.3)$ & & Reference \\
\hline Yes & 104 & $57(54.8)$ & $47(45.2)$ & & $4.108(2.376-7.102)$ \\
\hline IGRA & & & & $<0.001$ & \\
\hline Negative & 431 & $64(14.8)$ & $367(85.2)$ & & Reference \\
\hline Positive & 507 & 201 (39.6) & $306(60.4)$ & & $3.743(2.478-5.655)$ \\
\hline CD8+ & & & & 0.002 & \\
\hline$>500$ & 205 & $46(22.5)$ & $159(77.5)$ & & Reference \\
\hline$\leq 500$ & 468 & $153(32.7)$ & $315(67.3)$ & & $1.992(1.289-3.076)$ \\
\hline
\end{tabular}

$T B$ tuberculosis, BALF bronchoalveolar lavage fluid, NAAT nucleic acid amplification test, IGRA interferon- $\gamma$ release assays

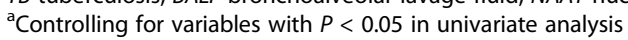


of $47.2 \%$ and $32.4 \%$, respectively. The overall sensitivity of BALF (63.4\%) was significantly higher than that of sputum (43.5\%) (see Table 2). Compared to sputum, an increased sensitivity of BALF was observed in all three Mtb tests including AFB staining, Mtb culture and NAAT, indicating that BALF samples were enriched for Mtb. Specifically, $122(19.7 \%)$ out of 620 pulmonary TB patients who were negative for all three Mtb tests in sputum were eventually confirmed by positive results for one or more of these tests in BALF. Notably, the benefit of BALF was mostly evident in those pulmonary TB suspects without sputum production. As shown in Table 3, 163 (40.0\%) out of 408 pulmonary TB patients without sputum production were bacteriologically confirmed by BALF Mtb tests.

\section{Factors associated with positive BALF Mtb detection in sputum-negative and non-sputum-producing pulmonary TB patients}

In line with previous literature [14], our data demonstrated that BALF significantly improved Mtb detection in pulmonary $\mathrm{TB}$ patients. More importantly, BALF significantly improved the diagnosis and treatment of those pulmonary TB patients who were unable to produce sputum. Isolating $\mathrm{Mtb}$ in those cases was not only critical for TB diagnosis, but also permitted drug susceptibility testing. Despite the clear benefit of bronchoscopy in the management of individual cases, only 285 (27.7\%) subjects were positive for Mtb detection in BALF among 1028 subjects with sputum negative or without sputum. To identify those patients most likely to benefit from bronchoscopy, we analyzed the clinical parameters associated with Mtb detection in BALF among sputumnegative pulmonary TB suspects and TB suspects who were unable to produce sputum. Univariate analysis showed that age $(P<0.001)$, hemoptysis $(P=0.002)$, bilateral lung infection $(P=0.005)$, cavity on chest radiographs $(P<0.001)$, CD4 cell count $(P=0.004)$, CD8 cell count $(P=0.001)$, erythrocyte sedimentation rate $(P=$ $0.001)$, and IGRA results $(P<0.001)$ were all significantly associated with Mtb detection in BALF (see Table 4). Multivariate logistic regression analysis revealed that age, cavity on chest radiographs (OR: 4.108, 95\% CI: $2.376-$

Table 6 Stratified analysis of factors associated with BALF positivity in pulmonary TB sputum-negative suspects and those without sputum

\begin{tabular}{|c|c|c|c|c|}
\hline & BALF $(+) n / N(\%)$ & BALF (-) n/N (\%) & $P$-value & $O R(95 \% C l)$ \\
\hline IGRA/Age & & & $<0.001$ & \\
\hline IGRA (-)/> 35 year & $30 / 239(12.6)$ & $209 / 239(87.4)$ & & Reference \\
\hline IGRA $(-) / \leq 35$ year & 34/192 (17.7) & 158/192 (82.3) & & $1.499(0.880-2.554)$ \\
\hline IGRA $(+) />35$ year & $55 / 212(25.9)$ & $157 / 212(74.1)$ & & $2.441(1.494-3.986)$ \\
\hline $\mid G R A(+) / \leq 35$ year & 146/295 (49.5) & $149 / 295(50.5)$ & & $6.826(4.372-10.658)$ \\
\hline Cavity/Age & & & $<0.001$ & \\
\hline Cavity $(-) />35$ year & 76/453 (17.0) & $371 / 477(83.0)$ & & Reference \\
\hline Cavity $(-) / \leq 35$ year & 152/477 (31.9) & $325 / 477(68.1)$ & & $2.283(1.669-3.122)$ \\
\hline Cavity $(+) />35$ year & $16 / 49(32.7)$ & $33 / 49(67.3)$ & & $2.367(1.240-4.516)$ \\
\hline Cavity $(+) / \leq 35$ year & $41 / 55(74.5)$ & $14 / 55(25.5)$ & & $14.296(7.426-27.521)$ \\
\hline CD8/Age & & & $<0.001$ & \\
\hline CD8> 500/> 35 year & $16 / 82(19.5)$ & $66 / 82(80.5)$ & & Reference \\
\hline CD8 $>500 / \leq 35$ year & $30 / 117(25.6)$ & $87 / 117(74.4)$ & & $1.422(0.716-2.824)$ \\
\hline$C D 8 \leq 500 />35$ year & 49/222 (22.1) & 173/222 (77.9) & & $1.168(0.621-2.197)$ \\
\hline$C D 8 \leq 500 / \leq 35$ year & $104 / 246(42.3)$ & $142 / 246(57.7)$ & & $3.021(1.655-5.515)$ \\
\hline IGRA/Cavity/Age & & & $<0.001$ & \\
\hline IGRA (-)/Cavity (-)/> 35 year & 24/219 (11.0) & $195 / 219(89.0)$ & & Reference \\
\hline IGRA (-)/Cavity $(-) / \leq 35$ year & 23/174 (13.2) & 151/174 (86.8) & & $1.238(0.672-2.278)$ \\
\hline IGRA (-)/Cavity $(+) />35$ year & $6 / 20(30.0)$ & $14 / 20(70.0)$ & & $3.482(1.223-9.912)$ \\
\hline IGRA (-)/Cavity $(+) / \leq 35$ year & $11 / 18(61.1)$ & 7/18 (38.9) & & $12.768(4.521-36.056)$ \\
\hline IGRA (+)/Cavity (-)/> 35 year & 45/184 (24.5) & 139/184 (75.5) & & $2.630(1.531-4.519)$ \\
\hline IGRA (+)/Cavity $(-) / \leq 35$ year & $118 / 262(45.0)$ & $144 / 262(55.0)$ & & $6.658(4.083-10.856)$ \\
\hline IGRA (+)/Cavity (+)/> 35 year & $10 / 28(35.7)$ & $18 / 28(64.3)$ & & $4.514(1.869-10.901)$ \\
\hline IGRA (+)/Cavity $(+) / \leq 35$ year & $28 / 33(84.8)$ & $5 / 33(15.2)$ & & $45.500(16.054-128.955)$ \\
\hline
\end{tabular}

TB tuberculosis, BALF bronchoalveolar lavage fluid, IGRA interferon- $\gamma$ release assays 
7.102), IGRA result (OR: $3.743,95 \% C I: 2.478-5.655)$, and CD8 counts (OR: $1.992,95 \%$ CI: 1.289-3.076) were independent risk factors associated with the positivity of Mtb detection in BALF (see Table 5). Further analysis indicated that combination of age, cavity on chest radiographs, and IGRA yielded the highest positivity of Mtb detection in BALF. Specifically, younger pulmonary TB suspects ( $\leq$ 35 years old) with cavity on chest radiographs and positive IGRA had $84.8 \%(28 / 33)$ positivity of Mtb detection in BALF (see Table 6).

\section{Discussion}

Negative diagnostic tests on sputum samples and lack of sputum production in patients with pulmonary TB have been reported to be the major causes of treatment delay [15]. In our study, the sensitivity of sputum Mtb tests including sputum Mtb culture and NAAT was 43.5\% (499/ $1146)$ in pulmonary TB patients. In paired comparison, $63.4 \%$ of $(727 / 1146)$ pulmonary TB patients were bacteriologically confirmed by BALF Mtb tests. In addition, 13 out of 25 patients with NTM infection were diagnosed by BALF-based tests. Thus, about $30 \%$ patients benefited from BALF Mtb tests. It is worthy to note that BALF Mtb tests increased $35.6 \%$ and $53.6 \%$ positivity in sputum-negative and non-sputum-producing pulmonary TB patients, respectively. BALF not only increased the positive rate of bacteriological detection for diagnosis, but also made a significant improvement for effective precision treatment through enabling species identification and drug susceptibility testing.

While bronchoscopy is clearly a useful diagnostic tool for pulmonary TB, it has risks of hemorrhage, pneumothorax, laryngospasm as well as other minor and major potentially adverse effects $[16,17]$. Nosocomial transmission of TB and other pathogens might also occur in some patients exposed to inadequately sterilized bronchoscopy equipment. Furthermore, bronchoscopy is an expensive procedure requiring expert personnel and facilities. Therefore, we identified clinical parameters associated with positivity of Mtb detection in BALF in an attempt to maximize the benefit of BALF collection for pulmonary TB suspects who undergo bronchoscopy. We found that age, cavity on chest radiographs, IGRAs results, and $\mathrm{CD}^{+} \mathrm{T}$ cell count are risk factors associated with positivity of BALF Mtb tests. By the stratified analysis, we determined that age, cavity on chest radiographs, IGRAs results, but not $\mathrm{CD}^{+} \mathrm{T}$ cell count, have an overlay effect in predicting positivity of Mtb tests in BALF. Specifically, a combination of age ( $\leq 35$ years), IGRAs $(+)$, cavity on chest radiographs had yielded a significantly high positivity (84.8\%) of Mtb detection in BALF.

Among these factors, cavity on radiograph has the highest predictive value, which might be expected, as the cavities developed by erosion of caseation granulomas into bronchi, through which Mtb can be expelled [18]. In support of this, a previous report indicated numerous AFB are detected at the surface of cavities [19]. In the present study, age $\leq 35$ years and positive IGRAs results were predictive factors for positive BALF results. Although IGRAs cannot reliably distinguish active TB from latent $\mathrm{TB}$ infection, we and other researchers have shown that the levels of IFN- $\gamma$ determined by IGRAs are associated with sputum Mtb positivity in immune competent pulmonary TB patients [20], and the sensitivity of IGRAs is correlated with paucibacillary nature of the samples [21]. In line with these findings, here we found IGRAs alone has a predictive odds ratio of 3.743 (95\% CI: $2.478-5.655$ ) for Mtb positivity in BALF, due to the existence Non-TB patients in the BALFnegative TB suspects. We speculate that younger patients capable of mounting a robust cell-mediated immune response to pulmonary $\mathrm{TB}$ are more likely to develop cavitary lesions with positive sputum and BALF tests for Mtb.

\section{Conclusions}

We found that age ( $\leq 35$ years), positive IGRA test and cavity on chest radiographs as risk factors associated with positivity of Mtb detection in BALF. Sputum negative pulmonary TB suspects who are under 35 years old, positive for the presence of pulmonary cavity and IGRA, should undergo bronchoscopy to collect BAFL for Mtb tests, as they have the highest possibility to get bacteriologically confirmation of TB.

\section{Additional file}

Additional file 1: Multilingual abstracts in the six official working languages of the United Nations. (PDF $714 \mathrm{~kb}$ )

\section{Abbreviations}

AFB: Acid fast bacilli; BALF: Bronchoalveolar lavage fluid; CDC: Center for Disease Control and Prevention; Cls: Confidence intervals; IGRAs: Interferongamma release assays; LPAs: Line probe assays; LTBI: Latent TB infection; MDR-TB: Multi-drug resistant TB; Mtb: Mycobacterium tuberculosis; NAAT: Nucleic acid amplification test; NTM: nontuberculous mycobacteria; ORs: Odds ratios

\section{Acknowledgements}

We would like to thank all study participants who were involved and contributed to the data collection. We thank editors and reviewers for their warm works and valuable comments.

\section{Funding}

This study was supported by National Nature Science Foundation of China (81525016, 81772145, 81770013), Shenzhen Science \& Technology Grant (JSGG20160427104724699, JCYJ20170412101048337, JCYJ20170412151620658), and Sanming Project of Medicine in Shenzhen (GCZX2015043015340574). 


\section{Availability of data and materials}

The datasets used and/or analysed during the current study are available from the corresponding author on reasonable request.

\section{Authors' contributions}

$\mathrm{XL}, \mathrm{X}-\mathrm{FH}, \mathrm{Q}-\mathrm{TY}, \mathrm{M}-\mathrm{XZ}, \mathrm{Z}-\mathrm{HW}$ and $\mathrm{H}-\mathrm{YL}$ performed the study. $\mathrm{XL}$ and $\mathrm{X}-\mathrm{FH}$ analyzed the data and wrote the manuscript. G-FD, Q-YD, T-SY and provided clinical data. LG helped the statistical analysis. HK helped edit the paper. B-PZ and X-CC supervised the study and applied for grants. XL and X-CC were identified as the guarantor of the paper, taking responsibility for the integrity of the work as a whole, from incepton to published article. All authors read and approved the final manuscript.

\section{Ethics approval and consent to participate}

The study was approved by the Institutional Review Board of Shenzhen Third People's Hospital, and informed consent was obtained from each participant.

\section{Consent for publication}

Not applicable.

\section{Competing interests}

The authors declare that they have no competing interests.

\section{Author details}

${ }^{1}$ Shenzhen Key Laboratory of Infection \& Immunity, Shenzhen Third People's Hospital (The Second Affiliated Hospital of Shenzhen University), Shenzhen University School of Medicine, Shenzhen, China. ${ }^{2}$ Department of Infectious Diseases, the Fifth Affiliated Hospital, Sun Yat-sen University, Zhuhai, China. ${ }^{3}$ Ministry of Health Key Laboratory of Systems Biology of Pathogens, Institute of Pathogen Biology, Chinese Academy of Medical Sciences and Peking Union Medical College, Beijing, China. ${ }^{4}$ Department of Medicine, University of Massachusetts Medical School, Worcester, MA 01655, USA. ${ }^{5}$ Yuebei Second People's Hospital, Shaoguan, China. 'Department of Pathogen Biology, Shenzhen University School of Medicine, Shenzhen 518054, China.

Received: 12 July 2017 Accepted: 12 March 2018

Published online: 24 March 2018

\section{References}

1. World Health Organization. Global tuberculosis report 2016. http://apps. who.int/medicinedocs/en/d/Js23098en/. Accessed 17 Dec 2017.

2. Horsburgh CJ, Barry CR, Lange C. Treatment of tuberculosis. N Engl J Med. 2015;373:22

3. Trebucq A, Devi S. Widespread use of Xpert((R)) MTB/ NAAT will most probably reduce the total number of TB cases notified. Int J Tuberc Lung Dis. 2015:19:11.

4. Luetkemeyer AF, Kendall MA, Wu X, Lourenco MC, Jentsch U, Swindells S, et al. Evaluation of two line probe assays for rapid detection of Mycobacterium tuberculosis, tuberculosis (TB) drug resistance, and non-TB mycobacteria in HIV-infected individuals with suspected TB. J Clin Microbiol. 2014;52:4.

5. Greco S, Girardi E, Navarra A, Saltini C. Current evidence on diagnostic accuracy of commercially based nucleic acid amplification tests for the diagnosis of pulmonary tuberculosis. Thorax. 2006;61:9.

6. Maynard-Smith L, Larke N, Peters JA, Lawn SD. Diagnostic accuracy of the Xpert MTB/RIF assay for extrapulmonary and pulmonary tuberculosis when testing non-respiratory samples: a systematic review. BMC Infect Dis. 2014;14:709.

7. George PM, Mehta M, Dhariwal J, Singanayagam A, Raphael CE, Salmasi M, et al. Post-bronchoscopy sputum: improving the diagnostic yield in smear negative pulmonary TB. Respir Med. 2011;105:11.

8. Tian P, Shen Y, Wang Y, Wan C, Feng M, Zhu J, et al. Diagnostic value of nucleic acid amplification tests on bronchoalveolar lavage fluid for smearnegative pulmonary tuberculosis: a meta-analysis. Biosci Rep. 2015;35:4.

9. Soto A, Acurio V, Solari L, Van der Stuyft P. Incremental yield of bronchial washing for diagnosing smear-negative pulmonary tuberculosis. Rev Saude Publica. 2013:47:4.

10. World Health Organization. Treatment of tuberculosis-guidelines for national programmes, fourth edition. 2010. http://www.who.int/tb/publications/ 9789241547833/en/. Accessed 17 Dec 2017.
11. Wang L, Zhang $H$, Ruan $Y$, Chin DP, Xia Y, Cheng S, et al. Tuberculosis prevalence in China, 1990-2010; a longitudinal analysis of national survey data. Lancet. 2014;383:9934.

12. Chegou NN, Sutherland JS, Malherbe S, Crampin AC, Corstjens PL, Geluk A, et al. Diagnostic performance of a seven-marker serum protein biosignature for the diagnosis of active TB disease in African primary healthcare clinic attendees with signs and symptoms suggestive of TB. Thorax. 2016;71:9.

13. Chen X, Yang Q, Zhang M, Graner M, Zhu X, Larmonier N, et al. Diagnosis of active tuberculosis in China using an in-house gamma interferon enzyme-linked immunospot assay. Clin Vaccine Immunol. 2009;16:6.

14. Chou PC, Wang CH, Huang CD, Lo YL, Lee KY, Yu CT, et al. Nucleic acid amplification test and bronchoscopy improve the diagnostic accuracy of smear-negative tuberculosis. Int J Tuberc Lung Dis. 2012;16:12.

15. Castro ATE, Mendes M, Freitas S, Roxo PC. Diagnostic yield of sputum microbiological analysis in the diagnosis of pulmonary tuberculosis in a period of 10 years. Rev Port Pneumol. 2015;21:4.

16. Ost DE, Ernst A, Lei X, Kovitz KL, Benzaquen S, Diaz-Mendoza J, et al. Diagnostic yield and complications of bronchoscopy for peripheral lung lesions. Results of the AQulRE registry. Am J Respir Crit Care Med. 2016;193:1.

17. Fernandez-Bussy S, Labarca G, Zagolin M, Oyonarte M, Isamit D, Jalilie A, et al. Immediate complications following flexible bronchoscopy: retrospective analysis of 1079 procedures. Rev Med Chil. 2014;142:3.

18. Hunter RL. On the pathogenesis of post primary tuberculosis: the role of bronchial obstruction in the pathogenesis of cavities. Tuberculosis (Edinb). 2011;91(Suppl 1):S6-10.

19. Kaplan G, Post FA, Moreira AL, Wainwright H, Kreiswirth BN, Tanverdi M, et al. Mycobacterium tuberculosis growth at the cavity surface: a microenvironment with failed immunity. Infect Immun. 2003;71:12.

20. De Keyser E, De Keyser F, De Baets F. Tuberculin skin test versus interferon-gamma release assays for the diagnosis of tuberculosis infection. Acta Clin Belg. 2014;69:5.

21. Liu F, Du FJ, Jia HY, Pan LP, Zhang $X$, Xing AY, et al. Inadequate values from an interferon-gamma release assay for smear-negative tuberculosis in a high-burden setting. Int J Tuberc Lung Dis. 2014;18:12.

\section{Submit your next manuscript to BioMed Central and we will help you at every step:}

- We accept pre-submission inquiries

- Our selector tool helps you to find the most relevant journal

- We provide round the clock customer support

- Convenient online submission

- Thorough peer review

- Inclusion in PubMed and all major indexing services

- Maximum visibility for your research

Submit your manuscript at www.biomedcentral.com/submit
) BioMed Central 